\title{
COMPORTAMENTO DA DORMÊNCIA EM SEMENTES DE Brachiaria dictyoneura cv. Llanero SUBMETIDAS ÀS AÇÕES DO CALOR E DO ÁCIDO SULFÚRICO ${ }^{1}$
}

\author{
CLODOALDO ROCHA DE ALMEIDA ${ }^{2}$; WALTER RODRIGUES DA SILVA ${ }^{3}$
}

\begin{abstract}
RESUMO - A literatura específica destaca a dormência das sementes como fator dificultador do estabelecimento das pastagens de gramíneas forrageiras tropicais. A imersão das sementes em ácido sulfúrico $\left(\mathrm{H}_{2} \mathrm{SO}_{4}\right)$, apesar de funcionalmente admitida como eficiente para promover a redução da dormência em sementes de braquiária, apresenta inconveniências relacionadas à segurança do trabalhador envolvido na aplicação e, adicionalmente, à preservação do meio ambiente com o descarte dos resíduos provenientes da operação. Dessa maneira, objetivando identificar alternativas para a superação da dormência, o presente estudo realizou comparações entre os efeitos fisiológicos de tratamentos térmicos $\left(55,65,75\right.$ e $85^{\circ} \mathrm{C} / 5,10$ e 15 h) e químico (imersão em $\mathrm{H}_{2} \mathrm{SO}_{4} / 15$ minutos) em sementes de Brachiaria dictyoneura cv. Llanero (Brachiaria humidicola cv. Llanero, segundo Renvoize et al., 1996). Para tanto, as sementes foram qualitativamente avaliadas (teor de água, germinação, tetrazólio, primeira contagem de germinação, emergência de plântulas, comprimento da parte aérea das plântulas e índice de velocidade de emergência) em duas épocas experimentais (início e final do armazenamento de 6 meses). Os usos do calor e do ácido sulfúrico constituem-se em alternativas para a redução da dormência das sementes e conseqüente favorecimento do desempenho agronômico. Contudo, particularmente em relação ao calor, a deterioração pode ser acelerada durante o armazenamento.
\end{abstract}

Termos para indexação: gramínea tropical, armazenamento, viabilidade.

\section{EFFECTS OF HEATING AND SULFURIC ACID ON SEED DORMANCY OF Brachiaria dictyoneura $\mathrm{cv}$. Llanero}

\begin{abstract}
Seed dormancy is emphasized in the literature as a problem for the establishment of tropical pasture grasses. Immersion of seeds in sulfuric acid $\left(\mathrm{H}_{2} \mathrm{SO}_{4}\right)$ has been widely used and is effective in breaking dormancy of Brachiaria seeds, although this treatment raises inconveniences relating to operator safety during application. Moreover, refusal of residues from this chemical treatment may become harmful to the environment. This study aims to evaluate alternative seed treatments for overcoming dormancy, comparing physiological effects from heating treatments $\left(55,65,75\right.$ and $85^{\circ} \mathrm{C}$, for 5,10 and 15 hour) and chemical scarification (immersion in $\mathrm{H}_{2} \mathrm{SO}_{4}$ for 15 minutes) in Brachiaria dyctioneura cv. Llanero seeds (Brachiaria humidicola cv. Llanero, according to Renvoize et al., 1996). Therefore, seeds were evaluated for their quality attributes (water content, germination, tetrazolium, first count of germination, seedling emergence, shoot length of seedlings, and emergence speed index), twice during the experiment (at the beginning and at the end of a six-month storage period). Heating and sulfuric acid are alternative treatments for overcoming seed dormancy, consequently favoring agronomic performance. However, particularly for the heating treatment, seed deterioration may be accelerated during storage.
\end{abstract}

Index terms: tropical grasses, storage, viability

\footnotetext{
${ }^{1}$ Submetido em 30/06/2003. Aceito para publicação em 29/01/2004. Parte da dissertação de Mestrado, do primeiro autor, apresentada à USP/ESALQ, Piracicaba,SP; trabalho conduzido com auxílio da FAPESP.

2 Engenheiro Agrônomo, Mestre em Agronomia. Rua Frederico Severo 12, 03574 030, São Paulo/SP. E-mail cra2@estadao.com.br.

3 Departamento de Produção Vegetal (USP/ESALQ), caixa postal 09, 13418-900, Piracicaba/SP. Bolsista do CNPq. E-mail wrsilva@carpa.ciagri.usp.br.
}

\section{INTRODUÇÃO}

Dentre os fatores que dificultam o uso das sementes de gramíneas forrageiras tropicais está a presença de dormência que, impedindo a germinação, interfere diretamente no estabelecimento uniforme da pastagem. Entende-se por dormência o fenômeno pelo qual as sementes, apesar de viáveis e dispondo das condições 
ambientais necessárias, não germinam (Carvalho \& Nakagawa, 1983). Nas gramíneas forrageiras tropicais, a expressão da dormência se associa a causas fisiológicas presentes em sementes recém-colhidas, progressivamente suprimidas durante o armazenamento, ou físicas, provavelmente relacionadas a restrições impostas pela cobertura da semente à entrada de oxigênio (Whiteman \& Mendra, 1982).

Métodos que visam a diminuição da dormência têm sido pesquisados, para usos laboratorial e industrial, empregando $\mathrm{H}_{2} \mathrm{SO}_{4}$ que, apesar de eficiente, pode gerar prejuízos aos trabalhadores operacionalmente envolvidos, à qualidade das sementes e ao meio ambiente. Segundo Oliveira \& Mastrocola (1983), o ácido sulfúrico interfere negativamente na germinação das sementes colhidas há mais de 10 meses; entretanto, Martins \& Lago (1996) verificaram que essa prática reduziu a dormência, sem prejuízos à qualidade, das sementes de Brachiaria brizantha armazenadas por 18 meses. A adequada eficiência do método, apesar de verificada em Brachiaria brizantha (Castro et al., 1994), foi menos evidente em $B$. decumbens (Atalla \& Toselo, 1979; González et al. 1994) e nula em $B$. humidicola (Atalla \& Toselo, 1979).

A utilização do calor tem sido, igualmente, investigada como método para a redução da dormência (Sharir, 1978). Exposições a 40 e $55^{\circ} \mathrm{C}$, durante 5 e 10 horas, proporcionaram ganhos fisiológicos às sementes de Panicum maximum (Martins \& Silva, 1998); em contrapartida, a temperatura de $85^{\circ} \mathrm{C}$ em períodos idênticos, apesar de reduzir a taxa de dormência, prejudicou fisiologicamente as sementes de Panicum maximum (Martins \& Silva, 1998) e de Brachiaria brizantha cv. Marandu (Martins \& Silva, 2001). Paralelamente, o emprego de $70^{\circ} \mathrm{C} / 10$ e 15 horas beneficiou o desempenho das sementes de $B$. brizantha, reduzindo a dormência sem gerar deterioração fisiológica latente (Martins \& Silva, 2001).

Assim, considerando a redução dos riscos, o tratamento térmico tem se apresentado como alternativa; contudo, existem dúvidas quanto à quantidade de calor, a ser usada nas diferentes espécies, para que seus efeitos se aproximem dos obtidos com o ácido.

O estado do conhecimento, em sementes de gramíneas forrageiras tropicais, não oferece segurança para orientar, de modo conclusivo, definições de procedimentos capazes de impedir a expressão da dormência no estabelecimento de pastagens. Desta maneira, estudos complementares, voltados à ampliação da base de informações sobre o tema, podem contribuir para alicerçar a formulação de alternativas tecnológicas que aliem, em sua aplicação, eficiência operacional e segurança ambiental. Assim, o presente trabalho teve o objetivo de avaliar os efeitos de tratamentos térmicos e do ácido sulfúrico no comportamento da dormência e no desempenho agronômico das sementes de Brachiaria dictyoneura cv. Llanero.

\section{MATERIAL E MÉTODOS}

Foram utilizados três lotes, de sementes de Brachiaria dictyoneura cv. Llanero (segundo Revoinze et al. (1996), Brachiaria humidicola cv. Llanero), provenientes de colheita de "varredura" com taxa de dormência igual ou superior a 43\%. Os lotes, após beneficiamento em máquina de ventilador e peneiras e em mesa gravitacional, passaram por seleção manual, para a retirada de impurezas, apresentando pureza física igual ou superior a $97 \%$.

Após o beneficiamento, as sementes foram submetidas a tratamentos térmicos com exposição durante 5,10 e 15 horas às temperaturas de $55,65,75$ e $85^{\circ} \mathrm{C}$ em estufa, e a tratamento de imersão em ácido sulfúrico $(98 \%$, $36 \mathrm{~N}$ ), por 15 minutos, seguida de lavagem em água corrente e secagem à sombra. Posteriormente, a reidratação líquida foi realizada para a uniformização do teor de água entre os tratamentos adotando, como referência, a umidade presente na testemunha (Martins \& Silva, 2001).

Após a obtenção dos tratamentos, foram conduzidas 2 épocas experimentais (início e final do armazenamento de 6 meses). A qualidade das sementes foi estimada através das determinações realizadas com 4 repetições e descritas a seguir.

a) Teor de água: foi avaliado a $105 \pm 3^{\circ} \mathrm{C} / 24 \mathrm{~h}$, pelo método da estufa (Brasil,1992), em duas amostras por repetição; os dados foram calculados com base no peso da matéria úmida (bu).

b) Germinação: o teste, com 50 sementes por repetição, foi conduzido em papel mata-borrão umedecido com água, em quantidade equivalente a 2,5 vezes o seu peso, sob temperaturas alternadas de $20-35^{\circ} \mathrm{C}$ (Brasil, 1992). As contagens foram feitas aos 7, 14 e 28 dias após a instalação do teste. Em seguida, as sementes não germinadas foram submetidas ao teste de tetrazólio para as identificações das taxas de sementes dormentes e mortas.

c) Teste de tetrazólio: as sementes remanescentes do teste de germinação, cortadas longitudinal e bilateralmente, foram imersas em solução aquosa $(0,5 \%)$ de 2,3,5 cloreto de trifenil-tetrazólio a $40^{\circ} \mathrm{C} / 4 \mathrm{~h}$ para, após identificação das sementes viáveis (dormentes) e mortas (Delouche et al., 1962), serem calculadas as taxas de dormência e de mortalidade em relação à população submetida ao teste de germinação.

d) Primeira contagem de germinação: foi realizada considerando a taxa (\%) de plântulas normais (Brasil, 1992) presente no $7^{\circ}$ dia após a semeadura no teste de germinação.

e) Emergência das plântulas: a semeadura (50 sementes/repetição) foi realizada a $0,5 \mathrm{~cm}$ de profundidade em substrato, de areia lavada, umedecido $(60 \%$ da capacidade de campo) com água destilada. O teste foi conduzido em condições ambientais não controladas de laboratório e, 14 dias após a instalação, foram contadas as 
plântulas que apresentaram a parte aérea exposta acima da superfície do substrato (Martins \& Silva, 2001).

f) Comprimento da parte aérea das plântulas: utilizando as plântulas emersas no teste de emergência, foi avaliado o comprimento (colo-ápice) da parte aérea; o valor $(\mathrm{mm})$ foi representado pelo quociente entre o somatório dos comprimentos obtidos e o total de sementes instaladas (Martins \& Silva, 2001).

g) Índice de velocidade de emergência: foi realizado utilizando o teste de emergência. O cálculo, estabelecido por Maguire (1962), considerou as contagens diárias das plântulas emersas entre o $5^{\circ}$ e o $14^{\circ}$ dias após a instalação do teste.

Foi utilizado delineamento em blocos (lotes) ao acaso e empregado o sistema de análise estatística para microcomputadores - SANEST (Zonta et al., 1984) com médias comparadas pelo teste de Tukey $(5 \%)$.

\section{RESULTADOS E DISCUSSÃO}

As variações entre os teores de água, surgidas após a aplicação dos tratamentos térmicos, foram adequadamente reduzidas pela reidratação (Tabela 1). Com isso, as avaliações fisiológicas puderam ser conduzidas sem interferências expressivas de diferenças, entre os tratamentos, devidas ao teor de água. Após o período de armazenamento, as variações no teor de água das sementes foram similares entre os tratamentos e, dessa maneira, indicaram a presença de uniformidade ambiental interna no local utilizado.

No início do armazenamento (Tabela 2), os tratamentos de $85^{\circ} \mathrm{C} / 5,10$ e $15 \mathrm{~h}$ e de $\mathrm{H}_{2} \mathrm{SO}_{4}$ reduziram, com diferença estatística, a taxa de sementes dormentes em relação à testemunha; os demais tratamentos, apesar de promoverem essa redução em valores absolutos na maioria dos casos, não apresentaram diferenciação estatística em relação à testemunha. Após o armazenamento de 6 meses, os tratamentos de $85^{\circ} \mathrm{C} / 10$ e $15 \mathrm{~h}$ e de $\mathrm{H}_{2} \mathrm{SO}_{4}$ mantiveram a inferioridade estatística em relação à testemunha (Tabela 3). Assim, de um modo geral, foi possível observar que a elevação no fornecimento de calor tendeu a diminuir o número de sementes dormentes de forma a permitir que os usos de $85^{\circ} \mathrm{C} / 10$ e $15 \mathrm{~h}$ apresentassem, estatisticamente, efeitos similares aos obtidos pelo $\mathrm{H}_{2} \mathrm{SO}_{4}$. A eficiência de tratamentos térmicos e de imersão em ácido sulfúrico, no que se refere à redução da taxa de dormência, foi similarmente constatada em sementes de Brachiaria decumbens (Castro et al., 1996) e B. brizantha (Martins et al., 1997).

A taxa de plântulas normais, no que se refere aos efeitos imediatos, permite verificar que os tratamentos de $65^{\circ} \mathrm{C} / 10$ e $15 \mathrm{~h}$, de $75^{\circ} \mathrm{C} / 5,10$ e $15 \mathrm{~h}$, de $85^{\circ} \mathrm{C} / 5,10$ e $15 \mathrm{~h}$ e de $\mathrm{H}_{2} \mathrm{SO}_{4}$ foram estatisticamente superiores à testemunha (Tabela 2). Contudo, após o armazenamento das sementes por 6 meses (Tabela 3), os tratamento não diferenciaram-se estatisticamente da testemunha; adicionalmente, considerando os valores absolutos, os de $85^{\circ} \mathrm{C} / 10$ e 15 horas foram inferiores a todos os demais.

TABELA 1. Teor (\%) de água nas sementes: dados obtidos nas situações seqüenciais A (antes do tratamento), B (após tratamento), C (após reidratação) e D (após armazenamento de 6 meses).

\begin{tabular}{cccccc}
\hline \multicolumn{2}{c}{ Tratamentos } & A & B & C & D \\
\hline \multicolumn{2}{l}{ Testemunha } & 13,03 & - & - & 12,66 \\
$55^{\circ} \mathrm{C} /$ & $5 \mathrm{~h}$ & 13,03 & 5,50 & 11,22 & 12,40 \\
& $10 \mathrm{~h}$ & 13,03 & 4,02 & 10,80 & 12,33 \\
& $15 \mathrm{~h}$ & 13,03 & 4,49 & 10,91 & 12,14 \\
$65^{\circ} \mathrm{C} /$ & $5 \mathrm{~h}$ & 13,03 & 3,95 & 11,06 & 12,23 \\
& $10 \mathrm{~h}$ & 13,03 & 2,46 & 11,19 & 12,57 \\
& $15 \mathrm{~h}$ & 13,03 & 2,25 & 11,26 & 11,73 \\
$75^{\circ} \mathrm{C} /$ & $5 \mathrm{~h}$ & 13,03 & 2,85 & 11,26 & 12,39 \\
& $10 \mathrm{~h}$ & 13,03 & 2,02 & 11,44 & 12,36 \\
& $15 \mathrm{~h}$ & 13,03 & 2,15 & 11,00 & 12,61 \\
$85^{\circ} \mathrm{C} /$ & $5 \mathrm{~h}$ & 13,03 & 2,01 & 10,55 & 12,51 \\
& $10 \mathrm{~h}$ & 13,03 & 1,32 & 11,25 & 12,48 \\
& $15 \mathrm{~h}$ & 13,03 & 1,44 & 11,67 & 12,27 \\
$\mathrm{H}_{2} \mathrm{SO}_{4}$ & & 13,03 & 13,75 & - & 12,87 \\
\hline
\end{tabular}

Assim, levando em conta os tratamentos mais favoráveis $\left(85^{\circ} \mathrm{C} / 5,10\right.$ e $15 \mathrm{~h}$ e $\left.\mathrm{H}_{2} \mathrm{SO}_{4}\right)$ à redução da dormência e à elevação da taxa de plântulas normais em seus efeitos imediatos (Tabela 2), observa-se que $85^{\circ} \mathrm{C} / 10 \mathrm{e}$ $15 \mathrm{~h}$ tenderam a gerar, em valores absolutos, efeitos latentes (Tabela 3) desfavoráveis à qualidade estimada pela taxa de plântulas normais, da mesma forma que o observado por Martins \& Silva (2001) em armazenamento de 9 meses.

As taxas de plântulas anormais e de sementes mortas, examinadas em associação, refletem os eventuais prejuízos fisiológicos promovidos pelos tratamentos. A análise da Tabela 2 permite verificar alguns efeitos imediatos negativos dos tratamentos, em valores absolutos, nas comparações com a testemunha; estatisticamente, contudo, os prejuízos ocorreram exclusivamente com o uso de $\mathrm{H}_{2} \mathrm{SO}_{4}$ para a taxa de plântulas anormais. Após o armazenamento (Tabela 3), essa mesma tendência foi observada para a taxa de plântulas anormais e, em relação à taxa de sementes mortas, os usos de $85^{\circ} \mathrm{C} / 10$ e $15 \mathrm{~h}$ apresentaram efeitos desfavoráveis, estatisticamente significativos, nas comparações com a testemunha. Prejuízos fisiológicos latentes após o armazenamento, relacionados com a mortalidade das sementes escarificadas com ácido sulfúrico, foram observados por Macedo et al. (1994), em sementes de B. humidicola, e por González et al. (1994), em sementes de B. decumbens. Dessa forma, foi 
possível verificar que alguns procedimentos capazes de favorecer a redução da dormência e a elevação imediata do desempenho agronômico podem, com o passar do tempo, contribuir para a deterioração das sementes.

Os dados dos testes de vigor, salvaguardadas as variações inerentes a cada uma das determinações, indicaram, de um modo geral, efeitos imediatos positivos dos tratamentos em relação à testemunha (Tabela 2); os tratamentos de $65^{\circ} \mathrm{C} / 15 \mathrm{~h}$, de $75^{\circ} \mathrm{C} / 15 \mathrm{~h}$, de $85^{\circ} \mathrm{C} / 10$ e $15 \mathrm{~h}$ e de $\mathrm{H}_{2} \mathrm{SO}_{4}$ destacaram-se dos demais ao apresentarem maior número de casos de superioridade estatística em relação à testemunha e, dentre estes, os de $85^{\circ} \mathrm{C} / 10$ e $15 \mathrm{~h}$ e de $\mathrm{H}_{2} \mathrm{SO}_{4}$ foram invariavelmente superiores à testemunha. Por outro lado, a análise dos efeitos latentes (Tabela 3) evidenciou, em valores absolutos, desvantagens das aplicações de $85^{\circ} \mathrm{C} / 10$ e $15 \mathrm{~h}$ nas comparações com a testemunha que, na maioria dos casos, foi superada pelo uso de $\mathrm{H}_{2} \mathrm{SO}_{4}$.

Dessa forma, os dados obtidos indicam que os usos de $85^{\circ} \mathrm{C} / 10$ e 15 h e de $\mathrm{H}_{2} \mathrm{SO}_{4}$, em seus efeitos imediatos, são equivalentes na redução da dormência e no favorecimento ao desempenho agronômico; contudo, contrariamente ao observado para o $\mathrm{H}_{2} \mathrm{SO}_{4}$, o uso do calor, conforme há aumento de temperatura, tende a acelerar a deterioração das sementes em decorrência de seus efeitos latentes.

TABELA 2. Dados obtidos nos testes de germinação (G), primeira contagem de germinação (PCG), comprimento da parte aérea das plântulas (CPA), emergência das plântulas (E) e índice de velocidade de emergência (IVE) sem o armazenamento (efeitos imediatos).

\begin{tabular}{|c|c|c|c|c|c|c|c|c|c|}
\hline \multirow{2}{*}{\multicolumn{2}{|c|}{ Tratamentos }} & \multicolumn{4}{|c|}{$\mathrm{G}(\%)$} & \multirow{2}{*}{$\begin{array}{c}\text { PCG } \\
(\%)\end{array}$} & \multirow{2}{*}{$\begin{array}{l}\mathrm{CPA} \\
(\mathrm{mm})\end{array}$} & \multirow{2}{*}{$\begin{array}{c}\mathrm{E} \\
(\%)\end{array}$} & \multirow[b]{2}{*}{ IVE } \\
\hline & & $\begin{array}{l}\text { sementes } \\
\text { dormentes }\end{array}$ & $\begin{array}{l}\text { plântulas } \\
\text { normais }\end{array}$ & $\begin{array}{l}\text { plântulas } \\
\text { anormais }\end{array}$ & $\begin{array}{c}\text { sementes } \\
\text { mortas }\end{array}$ & & & & \\
\hline \multicolumn{2}{|c|}{ Testemunha } & $65,33 \mathrm{a}$ & $13,00 \mathrm{~d}$ & $2,00 \mathrm{~b}$ & $19,66 \mathrm{a}$ & $7,00 \mathrm{e}$ & $0,42 \mathrm{~d}$ & $3,00 \mathrm{f}$ & $0,00 \mathrm{~d}$ \\
\hline \multirow[t]{3}{*}{$55^{\circ} \mathrm{C} /$} & $5 \mathrm{~h}$ & $65,67 \mathrm{a}$ & $12,00 \mathrm{~d}$ & $2,66 \mathrm{~b}$ & $19,50 \mathrm{a}$ & $7,00 \mathrm{e}$ & $0,25 \mathrm{~d}$ & $2,00 \mathrm{f}$ & $0,00 \mathrm{~d}$ \\
\hline & $10 \mathrm{~h}$ & $61,00 \mathrm{a}$ & $13,33 \mathrm{~d}$ & $2,50 \mathrm{~b}$ & $22,83 \mathrm{a}$ & $9,00 \mathrm{de}$ & $0,64 \mathrm{~d}$ & $4,33 \mathrm{def}$ & $0,00 \mathrm{~d}$ \\
\hline & $15 \mathrm{~h}$ & 64,17 a & $14,00 \mathrm{~d}$ & $1,83 \mathrm{~b}$ & $19,83 \mathrm{a}$ & 9,83 cde & $0,58 \mathrm{~d}$ & $3,83 \mathrm{ef}$ & $0,00 \mathrm{~d}$ \\
\hline \multirow[t]{3}{*}{$65^{\circ} \mathrm{C} /$} & $5 \mathrm{~h}$ & $62,50 \mathrm{a}$ & $16,33 \mathrm{~cd}$ & $1,00 \mathrm{~b}$ & $20,16 \mathrm{a}$ & $11,50 \mathrm{cde}$ & $1,00 \mathrm{~cd}$ & $5,00 \mathrm{def}$ & $0,00 \mathrm{~d}$ \\
\hline & $10 \mathrm{~h}$ & $49,00 \mathrm{ab}$ & $27,16 \mathrm{bc}$ & $2,33 \mathrm{~b}$ & 20,66 a & $21,50 a b c$ & $2,58 \mathrm{bcd}$ & 9,00 cdef & $0,16 \mathrm{~cd}$ \\
\hline & $15 \mathrm{~h}$ & $47,33 \mathrm{ab}$ & $29,66 \mathrm{~b}$ & $3,16 \mathrm{~b}$ & 19,66 a & $24,33 \mathrm{ab}$ & $4,92 \mathrm{ab}$ & $17,50 \mathrm{bc}$ & $0,66 \mathrm{abcd}$ \\
\hline \multirow[t]{3}{*}{$75^{\circ} \mathrm{C} /$} & $5 \mathrm{~h}$ & $45,00 \mathrm{ab}$ & $29,50 \mathrm{~b}$ & $3,00 \mathrm{~b}$ & $22,33 \mathrm{a}$ & $21,00 \mathrm{abcd}$ & $1,08 \mathrm{~cd}$ & $6,16 \mathrm{def}$ & $0,00 \mathrm{~d}$ \\
\hline & $10 \mathrm{~h}$ & $45,67 \mathrm{ab}$ & $31,00 \mathrm{~b}$ & $2,66 \mathrm{~b}$ & $21,16 \mathrm{a}$ & $26,33 \mathrm{ab}$ & 3,67 abcd & 12,33 bcde & $0,41 \mathrm{bcd}$ \\
\hline & $15 \mathrm{~h}$ & $43,83 a b$ & $33,08 \mathrm{ab}$ & $3,00 \mathrm{~b}$ & $19,33 \mathrm{a}$ & $24,50 \mathrm{ab}$ & $4,58 \mathrm{abc}$ & $16,33 \mathrm{bc}$ & $0,66 \mathrm{abcd}$ \\
\hline \multirow[t]{3}{*}{$85^{\circ} \mathrm{C} /$} & $5 \mathrm{~h}$ & $35,83 \mathrm{bc}$ & $30,50 \mathrm{~b}$ & $4,66 \mathrm{~b}$ & $28,16 \mathrm{a}$ & 18,83 bcde & 3,75 abcd & $13,16 \mathrm{bcd}$ & $0,25 \mathrm{~cd}$ \\
\hline & $10 \mathrm{~h}$ & $33,33 \mathrm{bc}$ & $37,33 \mathrm{ab}$ & $3,16 \mathrm{~b}$ & $26,33 \mathrm{a}$ & $30,66 \mathrm{ab}$ & $5,42 \mathrm{ab}$ & $19,00 \mathrm{ab}$ & $0,75 \mathrm{abc}$ \\
\hline & $15 \mathrm{~h}$ & $30,83 \mathrm{bc}$ & $34,00 \mathrm{ab}$ & $2,33 \mathrm{~b}$ & $32,50 \mathrm{a}$ & $23,66 \mathrm{ab}$ & $6,75 \mathrm{a}$ & $20,66 \mathrm{ab}$ & $1,00 \mathrm{ab}$ \\
\hline \multicolumn{2}{|c|}{$\mathrm{H}_{2} \mathrm{SO}_{4}$} & $16,67 \mathrm{c}$ & $44,50 \mathrm{a}$ & $12,83 \mathrm{a}$ & $24,66 \mathrm{a}$ & $32,33 \mathrm{a}$ & $7,25 \mathrm{a}$ & $27,33 \mathrm{a}$ & $1,33 \mathrm{a}$ \\
\hline
\end{tabular}

Médias seguidas por letras distintas, nas colunas, diferem entre si ao nível de significância de 5\% pelo teste de Tukey. 
TABELA 3. Dados obtidos nos testes de germinação (G), primeira contagem de germinação (PCG), comprimento da parte aérea das plântulas (CPA), emergência das plântulas (E) e índice de velocidade de emergência (IVE) com o armazenamento de 6 meses (efeitos latentes).

\begin{tabular}{|c|c|c|c|c|c|c|c|c|}
\hline \multirow[b]{2}{*}{ Tratamentos } & \multicolumn{4}{|c|}{$\mathrm{G}(\%)$} & \multirow{2}{*}{$\begin{array}{c}\text { PCG } \\
(\%)\end{array}$} & \multirow{2}{*}{$\begin{array}{l}\text { CPA } \\
(\mathrm{mm})\end{array}$} & \multirow{2}{*}{$\begin{array}{c}\mathrm{E} \\
(\%)\end{array}$} & \multirow[b]{2}{*}{ IVE } \\
\hline & $\begin{array}{c}\text { sementes } \\
\text { dormentes }\end{array}$ & $\begin{array}{c}\text { plântulas } \\
\text { normais }\end{array}$ & $\begin{array}{l}\text { plântulas } \\
\text { anormais }\end{array}$ & $\begin{array}{c}\text { sementes } \\
\text { mortas }\end{array}$ & & & & \\
\hline Testemunha & $45,67 \mathrm{a}$ & $23,67 \mathrm{abc}$ & $4,00 \mathrm{~b}$ & $26,50 \mathrm{~b}$ & $19,67 \mathrm{abcd}$ & $0,50 \mathrm{~b}$ & $4,67 \mathrm{bc}$ & $0,00 \mathrm{~b}$ \\
\hline $55^{\circ} \mathrm{C} / 5 \mathrm{~h}$ & $41,33 \mathrm{abc}$ & $26,00 \mathrm{abc}$ & $5,83 \mathrm{~b}$ & $26,83 \mathrm{~b}$ & $18,67 \mathrm{abcd}$ & $1,17 \mathrm{~b}$ & $7,33 \mathrm{bc}$ & $0,08 \mathrm{~b}$ \\
\hline $10 \mathrm{~h}$ & $43,33 \mathrm{ab}$ & $23,50 \mathrm{abc}$ & $6,50 \mathrm{~b}$ & $26,67 \mathrm{~b}$ & $17,00 \mathrm{abcd}$ & $1,08 \mathrm{~b}$ & $7,83 \mathrm{bc}$ & $0,08 \mathrm{~b}$ \\
\hline $15 \mathrm{~h}$ & $40,67 \mathrm{abc}$ & $22,17 \mathrm{abc}$ & $5,50 \mathrm{~b}$ & $31,67 \mathrm{~b}$ & $16,00 \mathrm{bcd}$ & $0,83 \mathrm{~b}$ & $7,17 \mathrm{bc}$ & $0,00 \mathrm{~b}$ \\
\hline $65^{\circ} \mathrm{C} / 5 \mathrm{~h}$ & $38,50 \mathrm{abc}$ & $26,17 \mathrm{abc}$ & $5,33 \mathrm{~b}$ & $30,00 \mathrm{~b}$ & $20,67 \mathrm{abcd}$ & $0,83 \mathrm{~b}$ & $7,67 \mathrm{bc}$ & $0,00 \mathrm{~b}$ \\
\hline $10 \mathrm{~h}$ & $33,67 \mathrm{abc}$ & $31,67 \mathrm{ab}$ & $5,67 \mathrm{~b}$ & $29,00 \mathrm{~b}$ & $28,33 \mathrm{abc}$ & $0,75 \mathrm{~b}$ & $6,50 \mathrm{bc}$ & $0,00 \mathrm{~b}$ \\
\hline $15 \mathrm{~h}$ & $29,50 \mathrm{abc}$ & $30,00 \mathrm{abc}$ & $8,33 \mathrm{~b}$ & $31,67 \mathrm{~b}$ & $25,17 \mathrm{abcd}$ & $1,42 \mathrm{~b}$ & $7,50 \mathrm{bc}$ & $0,08 \mathrm{~b}$ \\
\hline $75^{\circ} \mathrm{C} / 5 \mathrm{~h}$ & $34,50 \mathrm{abc}$ & $26,50 \mathrm{abc}$ & $6,67 \mathrm{~b}$ & $32,33 \mathrm{~b}$ & $22,83 \mathrm{abcd}$ & $0,83 \mathrm{~b}$ & $6,17 \mathrm{bc}$ & $0,00 \mathrm{~b}$ \\
\hline $10 \mathrm{~h}$ & $28,17 \mathrm{abcd}$ & $29,17 \mathrm{abc}$ & $7,83 \mathrm{~b}$ & $34,83 \mathrm{~b}$ & $26,33 \mathrm{abcd}$ & $1,33 \mathrm{~b}$ & $9,17 \mathrm{~b}$ & $0,00 \mathrm{~b}$ \\
\hline $15 \mathrm{~h}$ & $22,67 \mathrm{abcd}$ & $34,00 \mathrm{a}$ & $7,00 \mathrm{~b}$ & $36,33 \mathrm{ab}$ & $29,50 \mathrm{ab}$ & $0,50 \mathrm{~b}$ & $5,50 \mathrm{bc}$ & $0,00 \mathrm{~b}$ \\
\hline $85^{\circ} \mathrm{C} / 5 \mathrm{~h}$ & $20,67 \mathrm{abcd}$ & $23,83 \mathrm{abc}$ & $7,83 \mathrm{~b}$ & $47,67 \mathrm{ab}$ & $21,00 \mathrm{abcd}$ & $0,25 \mathrm{~b}$ & $2,83 \mathrm{bc}$ & $0,00 \mathrm{~b}$ \\
\hline $10 \mathrm{~h}$ & $14,83 \mathrm{~cd}$ & $12,33 \mathrm{bc}$ & $6,67 \mathrm{~b}$ & $62,17 \mathrm{a}$ & $14,67 \mathrm{~cd}$ & $0,25 \mathrm{~b}$ & $3,33 \mathrm{bc}$ & $0,00 \mathrm{~b}$ \\
\hline $15 \mathrm{~h}$ & $16,67 \mathrm{bcd}$ & $15,67 \mathrm{c}$ & $7,67 \mathrm{~b}$ & $60,00 \mathrm{a}$ & $13,67 d$ & $0,17 \mathrm{~b}$ & $2,67 \mathrm{c}$ & $0,00 \mathrm{~b}$ \\
\hline $\mathrm{H}_{2} \mathrm{SO}_{4}$ & $1,50 \mathrm{~d}$ & $37,50 \mathrm{a}$ & $17,67 \mathrm{a}$ & $43,33 \mathrm{ab}$ & $31,17 \mathrm{a}$ & $6,17 \mathrm{a}$ & $26,83 \mathrm{a}$ & $1,33 \mathrm{a}$ \\
\hline
\end{tabular}

Médias seguidas por letras distintas, nas colunas, diferem entre si ao nível de significância de 5\% pelo teste de Tukey.

\section{CONCLUSÃO}

Os usos do calor e do ácido sulfúrico constituem-se em alternativas para a redução da dormência das sementes e conseqüente favorecimento do desempenho agronômico. Contudo, particularmente em relação ao calor, a deterioração pode ser acelerada durante o armazenamento.

\section{REFERÊNCIAS}

ATALLA, L.M.P.; TOSELO, J. Observações sobre dormência em duas espécies de Brachiaria: B. decumbens e humidicola em condições de laboratório. Científica, Jaboticabal, v.7, n.3, p.353-355, 1979.

BRASIL. Ministério da Agricultura. Regras para análise de sementes, Brasília, SNDA/ DNDV / CLAV, 1992. 365p.

CARVALHO, N.M.; NAKAGAWA, J. Sementes: ciência, tecnologia e produção. 2.ed. Campinas: Fundação Cargill, 1983. 429p.
CASTRO, C.R.T.; CARVALHO, W.L.; REIS, F.P. Influência do tratamento com ácido sulfúrico na germinação de sementes de Brachiaria brizantha Stapt. Revista Ceres, Viçosa, v.41, n.236, p.451-458, 1994.

CASTRO, C.R.T.; CARVALHO, W.L.; REIS, F.P.; BRAGA FILHO, J. M. Superação da dormência tegumentar em sementes de Brachiaria decumbens Stapf. Revista Ceres, Viçosa, v.43, n.245, p.67-75, 1996.

DELOUCHE, J.C.; STILL, T.W.; RASPET, M.; LIENHARD, M. The tetrazolium test for seed viability. Mississipi: Mississipi Agricultural Experiment Station, 1962. 63p. (Tecnical Bulletin, 51).

GONZÁLEZ, Y.; MENDOZA, F.; TORRES, R. Efecto del almaceniamento y la escarificacion quimica y mecanica sobre las semillas de Brachiaria decumbens cv. Basilisk. Pastos y Forrajes, La Habana, v.17, n.35, p.35-43, 1994.

MACEDO, E.E.; GROTH, D.; LAGO, A. A. Efeito da escarificação com ácido sulfúrico na germinação de sementes de Brachiaria humidicola (Rendle) Schweick. 
Pesquisa Agropecuária Brasileira,Brasília, v.29, n.3, p. 455-460, 1994.

MAGUIRE, J.D. Speed of germination: aid in selection and evaluation for seedling emergence and vigor. Crop Science, Madison, v.2, n.2, p.176-177, 1962.

MARTINS, L.; LAGO, A.A. Germinação e viabilidade de sementes de Brachiaria brizantha (Hochst. ex A. Rich.) Staupf durante o armazenamento. Revista Brasileira de Sementes, Brasília, v.18, n.2, p.262-266, 1996.

MARTINS, C.C.; SILVA, W.R. Superação de dormência em sementes de Panicum maximum Jacq.: seleção de métodos para aplicação em escala industrial. Planta Daninha, Rio de Janeiro, v.16, n.2, p.77-84, 1998.

MARTINS, L.; SILVA, W.R. Estudo do comportamento da dormência em sementes de Brachiaria brizantha cultivar Marandu. Piracicaba, Pesquisa Agropecuária Brasileira, Brasília, v.36, n.7, p.997-1003, 2001.

MARTINS, L.; SILVA, W.R; LOT, R.C. Tratamentos térmicos e superação da dormência em sementes de Brachiaria brizantha (Hoechst. ex A. Rich) Stapf. Informativo ABRATES, Curitiba, v.7, n. 1 e 2, p.245,
1997.

OLIVEIRA, P.R.P.; MASTROCOLA, M.A. Brachiaria humidicola (Rendle) Schweick: observações acerca da viabilidade de suas sementes. Boletim de Indústria Animal, Nova Odessa, v.40, n.1, p.49-53, 1983.

RENVOIZE, S.A.; CLAYTON, W.D.; KABUYE, C.H.S. Morphology, taxonomy, and natural distribution of Brachiaria (Ed.) Griseb. In: MILES, J.W.; MAASS, B.L.; VALLE, C.B. do. Brachiaria: biology, agronomy, and improvement. Cali: CIAT,1996. p.1-15.

SHARIR, A. Some factors affecting dormancy breaking in plant seeds. Seed Science and Techonology, Zürich, v.6, n.3, p.655-660, 1978.

WHITEMAN, P.C.; MENDRA, K. Effects of storage and seed treatments on germination of Brachiaria decumbens. Seed Science and Technology, Zürich, v.10, p.233-242, 1982.

ZONTA, E.P.; MACHADO, A.A.; SILVEIRA JÚNIOR, P. Sistema de análise estatística para microcomputadores SANEST (software). Pelotas: UFPel, 1984. 